\title{
Non-Markovian dynamics of clusters during nucleation
}

\author{
J. Kuipers* \\ Institute for Theoretical Physics, Utrecht University, 3584 CE, Utrecht, The Netherlands \\ G. T. Barkema \\ Institute for Theoretical Physics, Utrecht University, 3584 CE, Utrecht, The Netherlands \\ and Institute-Lorentz for Theoretical Physics, Leiden University, 2333 CA, Leiden, The Netherlands
}

(Received 10 February 2009; revised manuscript received 2 April 2009; published 3 June 2009)

\begin{abstract}
Most theories of homogeneous nucleation are based on a Fokker-Planck-like description of the behavior of the mass of clusters. Here we will show that these approaches are incomplete for a large class of nucleating systems, as they assume the effective dynamics of the clusters to be Markovian, i.e., memoryless. We characterize these non-Markovian dynamics and show how this influences the dynamics of clusters during nucleation. Our results are validated by simulations of a three-dimensional Ising model with locally conserved magnetization.
\end{abstract}

DOI: 10.1103/PhysRevE.79.062101

PACS number(s): 64.60.qe

Nucleation is the process where a stable nucleus spontaneously emerges in a metastable environment. Excellent books and reviews exist on this topic; in his recent book, Kashchiev [1] listed about 30 books and 40 review articles on nucleation. Examples of nucleation abound, for instance, the formation of droplets in undercooled gasses and of crystals in undercooled liquids. The process is thermally activated and is a key to understanding various subjects in biophysics, polymer physics, and chemistry. The physics behind it has long been studied and the simplest version is known as the classical nucleation theory (CNT) [2,3].

In CNT the variations in the mass of a nucleus are described as a Markovian stochastic process in which single units attach and detach from the nucleus. The probability $p(m, t)$ that a nucleus has mass $m$ at time $t$ evolves via the Fokker-Planck equation $[3,4]$,

$$
\frac{\partial p(m, t)}{\partial t}=\frac{\partial}{\partial m}\left[R(m)\left(\beta \frac{\partial F}{\partial m}+\frac{\partial}{\partial m}\right) p(m, t)\right],
$$

with $R(m)$ as the rate at which clusters of mass $m$ grow to clusters of mass $m+1, F(m)$ is the free energy of a cluster of mass $m$, and $\beta$ is the inverse temperature. Phenomenological expressions for $R(m)$ and $F(m)$ are provided to complete the theory. $F(m)$ increases for small $m$ but decreases for large $m$, and it retains its maximal value at the so-called critical nucleus mass $m_{c}$. Therefore, clusters with mass below $m_{c}$ tend to shrink, whereas clusters larger than $m_{c}$ tend to grow. Starting at time $t=0$ with cluster mass $m(0)=m_{c}$, the mass evolves diffusively in time; its mean-square change, averaged over all realizations, $\left\langle\Delta m(t)^{2}\right\rangle \equiv\left\langle\left(m(t)-m_{c}\right)^{2}\right\rangle$, grows linearly with time for small deviations from the critical mass.

Although, qualitatively, there is ample experimental verification of the predictions of CNT; quantitatively, the nucleation rates it predicts may differ from experiment by 5-10 orders of magnitude [5]. CNT works really well if the nucleation process is correctly described by a Markov process

\footnotetext{
*jkuipers@phys.uu.nl
}

with units attaching and detaching as uncorrelated events. This is more or less the case in, for example, the Ising model with spin-flip dynamics. However, in the presence of a local conservation law, which, for example, is the case in binary mixtures of fluids or gasses and the Ising model with local spin-exchange dynamics, CNT shows serious shortcomings due to the neglect of memory effects by describing the cluster growth as a Markov process. The most dominant of these is usually the strong correlation in time between attachment and detachment events; after a particle detaches from the nucleus, it remains in its neighborhood for a relatively long time and is, therefore, likely to reattach. In fact, the theory of Brownian motion [6] states that in two dimensions, every detached particle will eventually return to the cluster, possibly after an extremely long time, and that in three dimensions, a fraction of the detached particles will never return. Figure 1 illustrates this memory effect in the Ising model with local spin-exchange dynamics. Two clusters of equal mass are shown. The cluster in the left panel has just shrunk, consequently has a higher density of particles surrounding it, which enhances the probability that the cluster will grow; in the right panel, the opposite is happening and the cluster is more likely to shrink. These memory effects result in a behavior quite different from CNT. The relation between the driving force $-\partial F / \partial m$ and the drift velocity is more complicated and the diffusion is anomalous, i.e., the mean-square displacement scales nonlinearly with time.

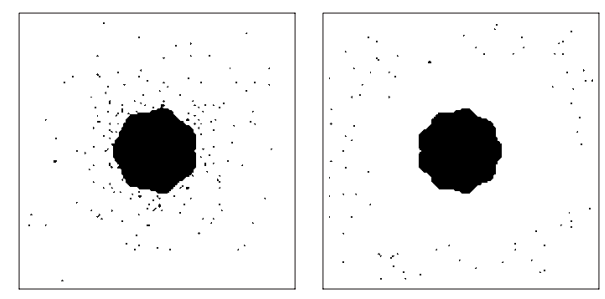

FIG. 1. Illustration of memory effects in the Ising model with local spin-exchange dynamics (exaggerated). Although they have equal masses, the cluster in the left panel is likely to grow as it has just shrunk, hence has higher density surrounding it, and vice versa in the right panel. 


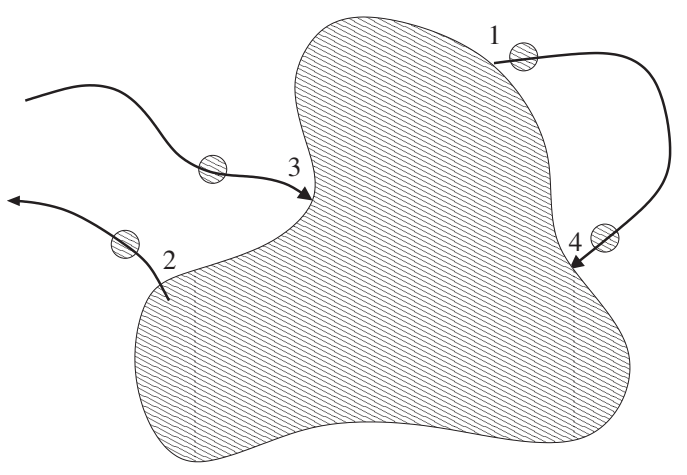

FIG. 2. The four effects causing variations in the cluster mass: (1) the emission of returning particles, (2) the emission the nonreturning particles, (3) the absorption of nonreturning particles, and (4) the absorption of returning particles.

We develop a theory for the variation in the cluster mass, where these memory effects are taken into account and show that on different time scales different types of dynamical behavior take place. We concentrate on states in threedimensional systems with a small gradient in free energy, which is the case, for instance, for near-critical nuclei. In that case, three distinct regimes show up. On very short time scales the mean-square growth of a cluster scales linearly with time similar to an ordinary diffusive process. This regime is followed by a regime of anomalous diffusion with an exponent of one half [i.e., $\left\langle\Delta m(t)^{2}\right\rangle \sim t^{1 / 2}$ ], and finally on large time scales linear growth occurs again, but with a much smaller prefactor than in the first regime. We validate our theory with extensive simulation results on the mean-square growth of near-critical nuclei in a three-dimensional Ising model with local spin-exchange dynamics.

In our model, we consider a cluster in a dilute environment (shown in Fig. 1). The variations in the cluster mass are caused by four effects, namely, (1) the emission of particles from the cluster that are returning (with return probability $\left.p_{r}\right)$, (2) the emission of nonreturning particles, (3) the absorption of particles from far away (i.e., not returning from previous emission), and (4) the absorption of particles that are returning. These four effects are illustrated in Fig. 2. The first three effects are independent and are described by random functions $\xi_{r}(t), \xi_{n r}(t)$, and $\xi_{n r}^{+}(t)$, respectively. Each of these random functions consists of a series of delta functions at Poisson distributed random times, so that the cluster mass $m(t)$ is an integer at all times. Their average values obey

$$
\left\langle\xi_{n r}^{+}(t)\right\rangle-\left\langle\xi_{n r}(t)\right\rangle=v(m(t)),
$$

with $v(m)$ as the systematic growth of a cluster of mass $m$. The variations $\delta \xi_{\alpha}(t) \equiv \xi_{\alpha}(t)-\left\langle\xi_{\alpha}(t)\right\rangle$ obey

$$
\sum_{ \pm}\left\langle\delta \xi_{n r}^{ \pm}(t) \delta \xi_{n r}^{ \pm}\left(t^{\prime}\right)\right\rangle=2\left(1-p_{r}\right) D_{M}(m(t)) \delta\left(t-t^{\prime}\right)
$$

and

$$
\left\langle\delta \xi_{r}(t) \delta \xi_{r}\left(t^{\prime}\right)\right\rangle=p_{r} D_{M}(m(t)) \delta\left(t-t^{\prime}\right),
$$

with $D_{M}(m)$ as the diffusion coefficient describing the shorttime mass variations in a cluster of mass $m$. Equations
(2)-(4) fully determine the three random functions $\xi_{\alpha}(t)$.

The absorption of returning particles is correlated with their emission; a returning particle emitted at time $\tau$ is returning at time $\tau+T_{\tau}$, with $T_{\tau}$ described by a return time distribution $\mu$. Putting this together, one may describe the evolution of a cluster by the stochastic differential equation,

$$
\dot{m}(t)=\xi_{n r}^{+}(t)-\xi_{n r}(t)-\xi_{r}(t)+\int_{-\infty}^{t} d \tau \delta\left(t-\tau-T_{\tau}\right) \xi_{r}(\tau) .
$$

The first two terms are basically CNT and the addition of the other two terms is new. This stochastic differential equation is too hard to be solved in general, and therefore we restrict ourselves first to near-critical clusters. On average critical clusters equally absorb many particles as they emit, so we assume $v(m)=0$. Furthermore, we assume that the diffusion coefficient is $m$ independent, $D_{M}(m)=D_{M}$. The average growth $\langle\Delta m(t)\rangle$ is then vanishing and the mean-square growth can be calculated from

$$
\left\langle\Delta m(t)^{2}\right\rangle=\int_{0}^{t} d \tau \int_{0}^{t} d \tau^{\prime}\left\langle\dot{m}(\tau) \dot{m}\left(\tau^{\prime}\right)\right\rangle,
$$

which, after substituting Eq. (5) twice and using Eqs. (3) and (4), results in the following expression:

$$
\left\langle\Delta m(t)^{2}\right\rangle=2 D_{M}\left[t-p_{r} \int_{0}^{t} d \tau(t-\tau) \mu(\tau)\right] .
$$

The first term in this equation is an ordinary diffusion, which is suppressed by the last term, since emission and absorption are correlated at larger time scales.

Next we investigate various limits of the equation. We assume that the time scale of spontaneous fluctuations (i.e., $\left.t \approx 1 / D_{M}\right)$ is much smaller than the time scale on which particles are typically returning. For small times the leading term in Eq. (7) results in

$$
\left\langle\Delta m(t)^{2}\right\rangle \approx 2 D_{M} t .
$$

At these time scales attachments and detachments occur at different places on the cluster surface as independent events, and therefore the mean-square cluster growth scales linearly with time.

To investigate $\left\langle\Delta m(t)^{2}\right\rangle$ at larger times we have to specify $\mu(t)$ in more detail. We assume that a detached particle makes a three-dimensional random walk with diffusion coefficient $D_{B}$. Furthermore, it starts at a distance $\delta R$ separated from the cluster, which we consider to be a sphere of radius $R$. This gives, for the return probability $p_{r}=\frac{R}{R+\delta R}$ and for the return time distribution [7],

$$
\mu(t)=\frac{\delta R \exp \left(-\delta R^{2} / 4 D_{B} t\right)}{\sqrt{4 \pi D_{B}} t^{3 / 2}} .
$$

Asymptotically at large times $\mu(t) \sim t^{-3 / 2}$, but it is cutoff at small times by the exponential. Using this distribution to calculate the mean-square cluster growth for larger times results in 

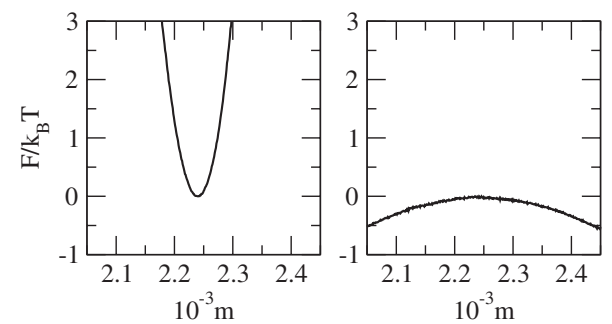

FIG. 3. Free-energy landscapes of a finite-sized Ising model, as a function of cluster mass: in the left panel with conserved magnetization and in the right panel without. The density in the left panel and the external magnetization in the right panel are tuned such that the extrema of the potentials coincide.

$$
\left\langle\Delta m(t)^{2}\right\rangle \approx 2\left(1-p_{r}\right) D_{M} t+\operatorname{const} \sqrt{t}
$$

At large time scales, the first term dominates, and the cluster dynamics is determined by particles being emitted to and absorbed from far away with rate $2\left(1-p_{r}\right) D_{M}$. At those large times, the change in cluster size is determined by nonreturning absorbed and emitted particles, and those particles can be treated as independent events. At intermediate time scales, however, the anomalous diffusion dominates if $p_{r}$ is close to 1 , which it typically is. This behavior is caused by emitted particles that are returning, much comparable to single file diffusion [8]. Note that if the return probability is 1 , the anomalous diffusion lasts forever. This may happen, for example, in many two-dimensional systems, although the longtime behavior is different from Eq. (10), due to a different return time distribution $\mu(t)$.

To validate the presented theoretical picture, simulations were performed on a three-dimensional Ising model with local spin-exchange (Kawasaki) dynamics with Metropolis acceptance probabilities $[9,10]$, a prototypic system to study nucleation. The Hamiltonian is given by

$$
H=-J \sum_{\langle i, j\rangle} s_{i} s_{j}+h \sum_{i} s_{i}
$$

with the first sum over all pairs of adjacent sites. A threedimensional cubic lattice of size $32 \times 32 \times 32$ with periodic boundary conditions has been used for the simulations. The temperature is chosen as $k_{B} T=2.5 \mathrm{~J}$, well below the critical temperature of $k_{B} T_{c} \approx 4.5 \mathrm{~J}$ [10]. An oversaturated initial configuration is first brought into equilibrium under constant magnetization, resulting in a single large cluster of $m_{c}$ spins in equilibrium with its surroundings with a density of $1.2 \%$. Next, besides the spin-exchange moves, we also perform spin flips in three strips of the box (one in each principal direction), as far away from the cluster as possible. These spin-flip moves mimic an infinite reservoir of up-pointing spins and render the cluster instable. The strength of the external field $h$ is fixed at the value giving the initial cluster a critical size, hence zero average growth rate. The free energy as a function of cluster mass is plotted in Fig. 3, before (left) and after (right) lifting the constraint on the magnetization.

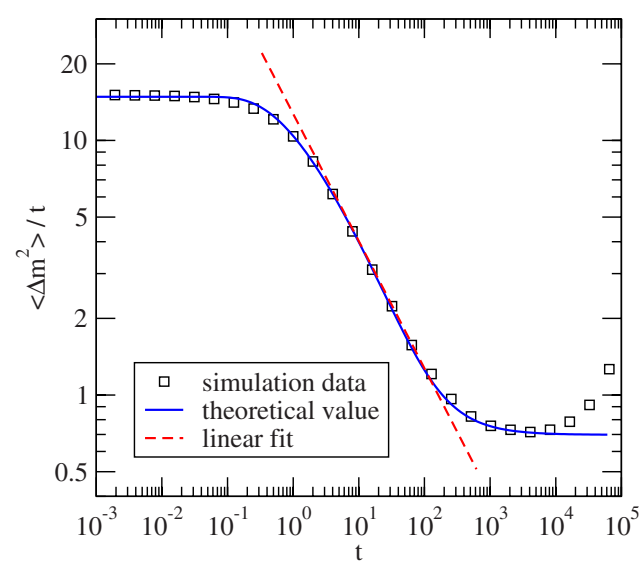

FIG. 4. (Color online) Measurements of $\left\langle\Delta m(t)^{2}\right\rangle / t$ in the Ising model plotted together with the theoretical value of Eq. (7) and a linear fit with a slope of minus one half. A zero slope indicates ordinary diffusion.

The time evolution of this cluster is then measured and the resulting mean-square change in cluster mass as a function of time, averaged over about 70,000 realizations, is shown in Fig. 4. For comparison, our theoretical estimate of $\left\langle\Delta m(t)^{2}\right\rangle$ is plotted with the simulation data. Our theory requires as input the function $\mu(t)$, with its parameters $D_{B}, R$, and $\delta R$, and the parameters $D_{M}$ and $p_{r}$. They are chosen as follows. The distribution $\mu(t)$ is as in Eq. (9), multiplied by an exponential, since the system has a finite size and long return times are exponentially suppressed. The result depends very little on the chosen exponent. Furthermore, $D_{B}$ $=\frac{1}{2}$, in accordance with the definition of time in our model, and $R$ is chosen such that $m_{c}=\frac{4}{3} \pi R^{3}$. The parameter $\delta R$ is the initial distance between the cluster and a spin which has just detached from it. If the spin detaches radially, this distance equals the lattice spacing, but the effective distance can be smaller by a factor of up to 2 in other directions. We chose $\delta R=\frac{8+\pi^{2}}{8 \pi}$, since that is the average distance between a spherical cluster and the sites neighboring it on the square lattice. The diffusion coefficient $D_{M}$ in Eq. (7) and subsequently the return probability $p_{r}$ are fitted.

The theory captures the general trend well. For short times ordinary diffusion is observed in the simulation data, after which anomalous diffusion with an exponent of one half is clearly present, as indicated by the dashed line in Fig. 4. For large times, however, the cluster is so far out of equilibrium that the gradient in the free energy is nonzero, so that $\langle\Delta m(t)\rangle \neq 0$ and $\left\langle\Delta m(t)^{2}\right\rangle$ grows superlinearly with time. We verified that the same results hold, qualitatively, for different values of the temperature.

Next, we turn to the consequences of these memory effects on nucleation times. To obtain nucleation times, Eq. (5) should be solved in the presence of a free-energy barrier, which results in a systematic growth $v(m)$ in Eq. (2). The phenomenological expression for the free energy from CNT $[2,3]$ or quadratic approximation could be used. This work is still in progress, and therefore we resort to scaling arguments in the meantime.

Instead of the nucleation time, we focus on the vaporization time (i.e., the time it takes for a critical nucleus to va- 
porize); these two times are connected via detailed balance by $T_{\text {nucl }} \approx e^{\beta \Delta F} T_{\text {vap }}$; hence $T_{\text {vap }}$ is the pre-exponential factor of the nucleation time. This vaporization time is mainly dictated by the time a critical cluster resides in a region near the top of the free-energy barrier; after that it vaporizes relatively fast due to the gradient in the free energy. We define this region near the top as the region where the free energy is less than $k_{B} T$ below the maximum value $F\left(m_{c}\right)$ and call its width $\delta m$. In CNT the residence time for this region, often referred to as the Zeldovich factor [11], scales as $T_{\text {res }} \sim \delta m^{2}$. If, in Eq. (10), the anomalous diffusion is taken over by the normal diffusion at the residence time, the memory effects only result in rescaling the diffusion coefficient by a factor of $1-p_{r}$. However, if the cluster dynamics show subdiffusive behavior up to this residence time, then $\left\langle\Delta m(t)^{2}\right\rangle \sim \sqrt{t}$, so that the residence time is proportional to $\delta m^{4}$. Which behavior occurs depends on numerous variables, such as the shape of the free-energy landscape and the mobility of detached particles.

Another view on the consequences of memory effects on the nucleation time is that compared to $\mathrm{CNT}$ with rescaled diffusion coefficient $D_{\text {eff }}=\left(1-p_{r}\right) D_{M}$, anomalous diffusion is present in addition to CNT's ordinary diffusion. This results in increased mass fluctuations around the critical mass, hence decreases the residence time and, therefore, also the nucleation time.

In summary, we have demonstrated that memory effects are playing an important role in the dynamics of nucleation. The time evolution of nucleating clusters shows consequently anomalous diffusion. These memory effects can be captured by a simple stochastic differential Eq. (5), which gives measurable results for critical clusters. These effects probably also have impact on the nucleation times. An analytic treatment of this equation in the presence of a freeenergy barrier is, therefore, an interesting topic for future research and might lead to new quantitative predictions for nucleation times.

We thank Henk van Beijeren for useful discussion.
[1] D. Kashchiev, Nucleation: Basic Theory with Applications (Butterworth-Heinemann, Oxford, 2000).

[2] R. Becker and W. Döring, Ann. Phys. 24, 719 (1935).

[3] J. D. Gunton and M. Droz, Introduction to the Theory of Metastable and Unstable States (Springer-Verlag, New York, 1983).

[4] N. G. van Kampen, Stochastic Processes in Physics and Chemistry (Elsevier, Amsterdam, 1981).

[5] S. Auer and D. Frenkel, Nature (London) 409, 1020 (2001).

[6] Z. Schuss, Theory and Applications of Stochastic Differential
Equations (Wiley, New York, 1980).

[7] The Laplace transform of this result can be found in J. G. Wendel, Ann. Probab. 8, 164 (1980).

[8] K. W. Kehr, R. Kutner, and K. Binder, Phys. Rev. B 23, 4931 (1981).

[9] K. Kawasaki, Phys. Rev. 145, 224 (1966).

[10] M. E. J. Newman and G. T. Barkema, Monte Carlo Methods in Statistical Physics (Oxford University Press, Oxford, 1999).

[11] Ya. B. Zel'dovich, Sov. Phys. JETP 12, 525 (1942). 